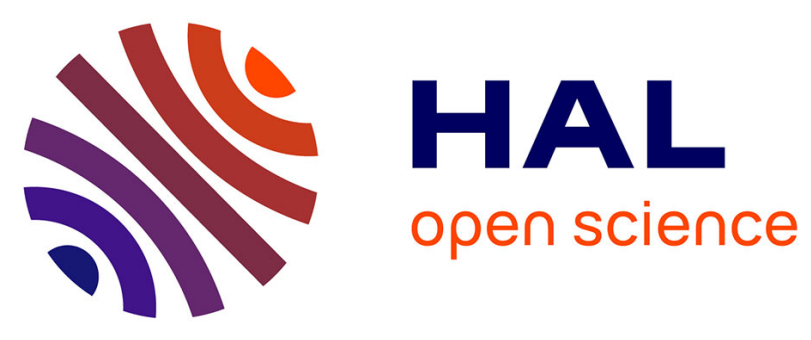

\title{
Extended aging of Ge-Se glasses below the glass transition temperature
}

Ellyn A. King, Sabyasachi Sen, Wataru Takeda, Catherine Boussard-Plédel, Bruno Bureau, Jean-Pierre Guin, Pierre Lucas

\section{- To cite this version:}

Ellyn A. King, Sabyasachi Sen, Wataru Takeda, Catherine Boussard-Plédel, Bruno Bureau, et al.. Extended aging of Ge-Se glasses below the glass transition temperature. Journal of Chemical Physics, 2021, 154 (16), 10.1063/5.0050474 . hal-03244928

\section{HAL Id: hal-03244928 \\ https://hal.science/hal-03244928}

Submitted on 13 Oct 2021

HAL is a multi-disciplinary open access archive for the deposit and dissemination of scientific research documents, whether they are published or not. The documents may come from teaching and research institutions in France or abroad, or from public or private research centers.
L'archive ouverte pluridisciplinaire HAL, est destinée au dépôt et à la diffusion de documents scientifiques de niveau recherche, publiés ou non, émanant des établissements d'enseignement et de recherche français ou étrangers, des laboratoires publics ou privés. 


\title{
Extended aging of Ge-Se glasses below the glass transition temperature.
}

\author{
Ellyn A. King ${ }^{1,2, \dagger}$, Sabyasachi Sen ${ }^{3}$, Wataru Takeda ${ }^{1}$, Catherine Boussard-Pledel ${ }^{2}$, Bruno \\ Bureau $^{2}$, Jean-Pierre Guin ${ }^{4}$, Pierre Lucas ${ }^{1, *}$ \\ ${ }^{1}$ Department of Materials Science and Engineering, University of Arizona, Tucson, Arizona 85721, USA \\ ${ }^{2}$ Univ Rennes, CNRS, ISCR (Institut des Sciences Chimiques de Rennes) - UMR 6226, F-35000 Rennes, \\ France \\ ${ }^{3}$ Department of Materials Science and Engineering, University of California, Davis, California 95616, \\ USA \\ ${ }^{4}$ Univ Rennes, CNRS, IPR (Institut de Physique de Rennes) - UMR 6251, Rennes, France \\ *Author to whom correspondence should be addressed: pierre@arizona.edu \\ ${ }^{\dagger}$ Now at Corning Inc, Corning Research and Development Corporation, Painted Post NY 14870
}

\begin{abstract}
Germanium selenide glasses of compositions spanning the whole glass-formation range are aged at room temperature for up to twenty years. A prominent enthalpy relaxation process is observed in all glasses and its structural origin is analyzed by Raman spectroscopy. The structural relaxation is manifested in the Raman spectra as a decrease in the ratio of edge to corner-sharing $\mathrm{GeSe}_{4 / 2}$ tetrahedral units. This structural evolution can be explained in terms of configurational entropy and density changes. Changes in Raman features and enthalpy follow an identical stretched exponential relaxation function characteristic of ageing in glasses. The compositional dependence of enthalpy relaxation after twenty years is in agreement with kinetic considerations based on the glass transition temperature of each glass. The relaxation behavior and heat-capacity curves are consistent with standard glass relaxation models for all compositions. These results indicate that the non-reversing enthalpy obtained by modulated differential scanning calorimetry (MDSC) which suggests the existence of non-aging glasses is not a reliable measure of the ability of a glass to relax. Instead, it is suggested that an interpretation of MDSC data in terms of complex heat capacity provides a more complete and reliable assessment of the relaxation properties of glasses.
\end{abstract}

\section{INTRODUCTION}

Sub- $T_{g}$ relaxation is an important problem in glass technology due to the unwanted property changes that can take place in glassy components over time. Volumetric changes are detrimental for glass substrates used in consumer electronics ${ }^{1}$ while changes in refractive index are harmful 
for optical applications ${ }^{2}$. Chalcogenide glasses are particularly exposed to these issues due to their relatively low glass transition temperature $\left(T_{g}\right)$ which can lead to significant relaxation even at room temperature ${ }^{3,4}$. Among these amorphous chalcogenides, germanium selenide glasses are of interest due to their complex structural dynamic ${ }^{5-7}$ and their precedent as testbeds for topological constraint theories 8,9 . The structure of Ge-Se glasses is composed of Se chains and $\mathrm{GeSe}_{4}$ tetrahedra that exhibit notably distinct dynamics as shown by Nuclear Magnetic Resonance (NMR) $)^{5-7}$. These two structural elements can in turn be related to the floppy and rigid domains that constitute the basis of topological constraint theories ${ }^{8,10,11}$. Indeed, Ge-Se glasses have long been used to test topological constraint theories due to their covalent network structure. The well-defined number of bonds and bond angles provides a convenient testbed for constraint counting principles which are thought to help predict some physical properties of the glass. For example, it has been observed that some properties such as density show a broad maximum near the so-called rigidity percolation threshold where the number of atomic degrees of freedom $(d=3)$ is exactly compensated by angular and bond constraints ${ }^{12,13}$. In this region the glass network is expected to undergo a floppy (under-coordinated)-to-rigid (over-coordinated) transition. Constraint counting shows that this transition should occur when the average coordination number (average number of bonds per atom) is $\langle r\rangle=2.4^{11}$.

More recently this theory has been extended to suggest that covalent networks can be optimally constrained over a broader compositional region where they are stress-free ${ }^{14}$. Experimental observation of this compositional window has been claimed mainly by invoking a quantity called the non-reversing enthalpy $\Delta H_{n r}$ measured by Modulated Scanning Differential Calorimetry (MDSC) ${ }^{9,15-17}$. In MDSC, the sample is subjected to a linear heating ramp superimposed with a sinusoidal oscillation. The resultant heat flow is often decomposed into a "reversing" heat capacity component and a "non-reversing" kinetic component permitting to separate nonreversible processes such as cold crystallization from the total heat flow ${ }^{18}$. This method was in turn applied to analysis of the glass transition with the goal of characterizing relaxation processes $^{19}$. In chalcogenide systems, it was observed that the non-reversing enthalpy decreases to zero in certain compositional ranges $(0.20$ to $0.25 \mathrm{Ge} \%$ in $\mathrm{Ge}-\mathrm{Se})$; thus, dubbed "reversibility window". It was further suggested that the self-organized nature of the network within this window would lead to glasses that do not age $\mathrm{e}^{9,15-17}$. This observation was derived from measurements of $\Delta H_{n r}$ on glasses annealed at room temperature for up to several weeks. It was found that chalcogen-rich glasses exhibited significant change in $\Delta H_{n r}$, while glasses in the "reversibility window" with lower chalcogen content exhibited minimal or no change ${ }^{9,15-17}$. However, one issue with this interpretation is that glasses with broadly different $T_{g}$ could have relaxation times spanning multiple orders of magnitude since the enthalpy relaxation time is exponentially dependent on $T_{g}{ }^{20,21}$. Hence, annealing series of glasses with different $T_{g}$ at a common temperature for the same duration cannot provide a systematic measure of their ability to relax but merely a broad assessment of their relative relaxation times. In other words, low- $T_{g}$ glasses may relax within a few weeks even at room temperature while relaxation may be too slow to observe for high- $T_{g}$ glasses within the "reversibility window". Therefore, more extensive 
analyses are needed to assess the ability of glasses from the so-called "reversibility window" to undergo aging.

In this study we analyze glasses across the floppy to rigid range that have been aged over 20 years at room temperature. Furthermore we analyze the time dependent relaxation of two glasses, one outside and one inside the "reversibility window", for up to five years at room temperature. It is found that all glass compositions exhibit significant relaxation in a way consistent with their relative $T_{g}$. Glasses outside and inside the "reversibility window" undergo a large stretchedexponential enthalpy relaxation characteristic of traditional glassy systems. Furthermore, the structural origin of this relaxation process is found to be identical in all glasses and shown to involve a conversion from edge to corner-sharing tetrahedral units that is consistent with a decrease in configurational entropy and an increase in density, as expected from conventional glass relaxation.

\section{BACKGROUND}

Sub- $T_{g}$ glass relaxation - also called aging - has been extensively studied over the years ${ }^{22-34}$ and it has been shown that the relaxation process depends on multiple factors including the nonexponentiality $\beta$, the non-linearity $x$, the fictive temperature $T_{f}$ and the fragility index $m$. The fragility is of particular interest as it affects the relaxation process in multiple ways that may appear contradictory. For example glasses located within the "reversibility window" are generally found to correspond to compositions exhibiting lower fragility index ${ }^{35}$ so that prior suggestions that these glasses do not age, may appear to indicate that low fragility is associated with a resistance to relaxation. On the other hand, recent energy landscape simulations have shown that high-fragility glasses are more resistant to relaxation during linear heat treatments due to the higher free energy barriers below the glass transition ${ }^{32}$. This apparent contradiction stems from the fact that fragility affects the relaxation process in two distinct ways: (i) higher fragility increases the thermodynamic driving force for relaxation and leads to greater relaxed enthalpy, (ii) higher fragility increases the relaxation time and leads to slower relaxation process.

The first case can be illustrated using a configurational entropy plot as in Fig. 1. As demonstrated by Martinez \& Angell ${ }^{36}$, the scaled entropy of glass-forming liquids follows the same pattern as the viscosity on a fragility plot and constitute a thermodynamic signature of fragility. The scaled entropy of three hypothetical glass-formers is therefore plotted in Fig. 1(a) using a modified Vogel-Tamman-Fulcher equation with fragility index $m=16,55$ and 200. The equilibrium lines are extended below $T_{g}$ while the glassy state is represented by the thick dashed line where excess entropy is frozen in. The part of the curve below $T_{g}$ is of particular interest here and is replotted in Fig. 1(b) as the normalized entropy versus normal temperature in a way similar to a Kauzmann plot $^{37}$ but normalized by $T_{g}$. It is clear from Fig. 1(b) that the entropy of the fragile glass diverges much more rapidly from the equilibrium line than the strong one. Consequently, a much greater thermodynamic driving force for relaxation builds up, while that of the strong 
system remains minimal for an equivalent departure from $T_{g}$. This indicates that fragile systems are prone to greater structural relaxation, once sufficient time is allowed to reach equilibrium.

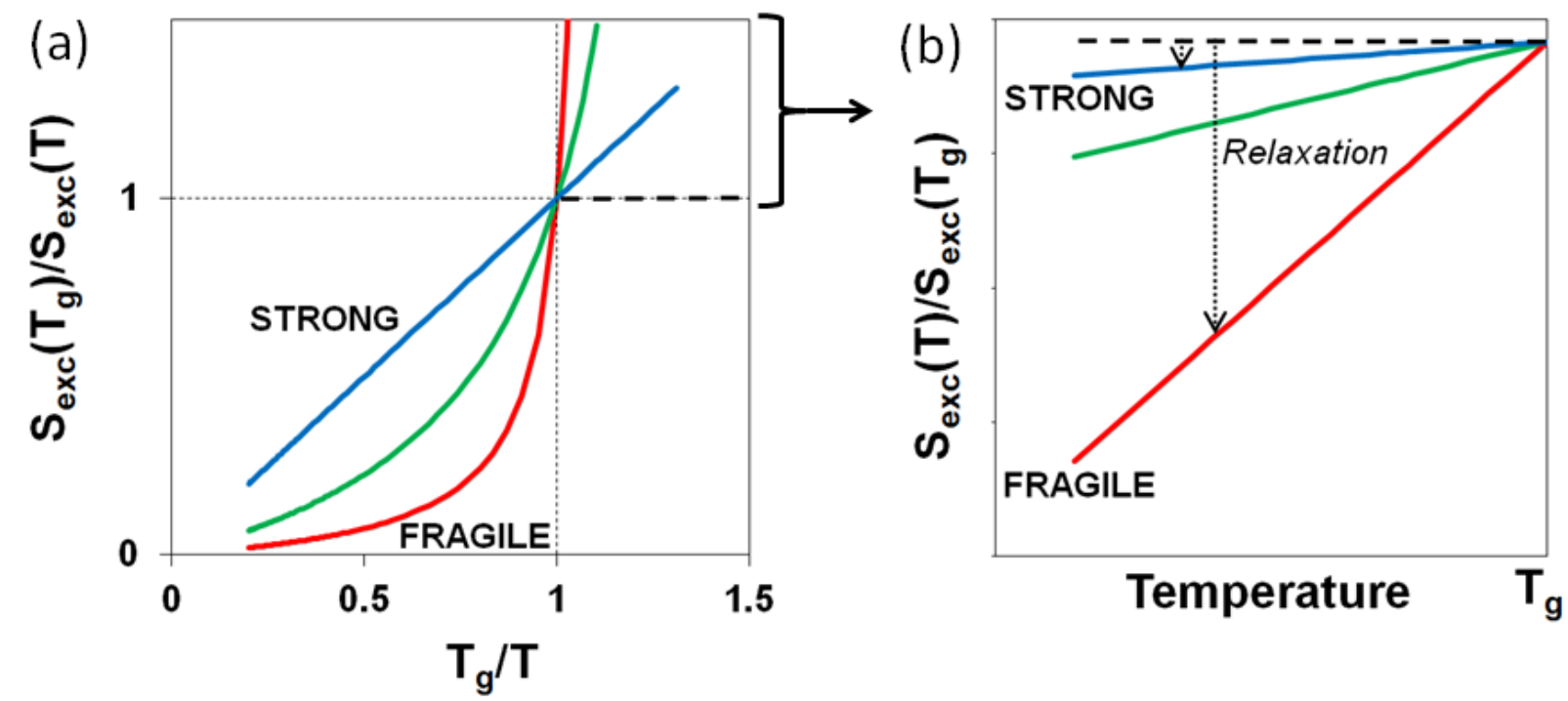

Fig. 1: (a) Scaled entropy (inverse excess entropy normalized by its value at $T_{g}$ ) for three hypothetical glass-formers with fragility ranging from $m=16$ to 200. Thick dashed lines indicate the idealized glass state where configurational entropy is frozen in. (b) Variation of the equilibrium entropy with temperature relative to the glassy state for the same three glass-formers.

The second case can be simply derived from the Tool-Narayanaswamy-Moynihan (TNM) equation for the structural relaxation time $\tau^{20,25}$ and the equation for the fragility index $m^{38}$ where the activation energy for enthalpy relaxation $\Delta H^{*}$ is considered equivalent to the activation for viscous flow as suggested by Moynihan ${ }^{39}$.

$$
\begin{gathered}
\tau=A \exp \left[\frac{x \Delta H^{*}}{R T}+\frac{(1-x) \Delta H^{*}}{R T_{f}}\right] \\
m=\frac{\Delta H^{*}}{R T_{g} \cdot \ln 10}
\end{gathered}
$$

It is then obvious that when the fragility index $m$ increases, the relaxation time $\tau$ increases exponentially. Therefore, when all other parameters are kept constant, relaxation is considerably slower in the fragile systems than in the strong ones.

In summary, fragile glasses have a greater thermodynamic potential for relaxation during aging, but their relaxation rate is much slower compared to that of the strong ones. 


\section{EXPERIMENT}

\section{A. Glass samples:}

Two sets of Ge-Se glasses were produced for this study. All Ge-Se samples were high purity glasses used for the preparation of optical elements. Syntheses were performed in evacuated silica tubes in a rocking furnace as detailed in ref $\left[{ }^{40}\right]$ and $\left[{ }^{41}\right]$. As shown in ref $\left[{ }^{41}\right]$ these glasses were fully homogenized and were free of impurities such as water, which may affect their structural and dynamic behavior as implied in ref [ $\left.{ }^{9}\right]$. The first set was a series of bulk glasses produced in 1999 with composition $\mathrm{Ge}_{10} \mathrm{Se}_{90}, \mathrm{Ge}_{15} \mathrm{Se}_{85}, \mathrm{Ge}_{20} \mathrm{Se}_{80}, \mathrm{Ge}_{25} \mathrm{Se}_{75}$ and $\mathrm{Ge}_{30} \mathrm{Se}_{70}{ }^{40,42}$. This set of glass was stored in air in a thermostated room $\left(22^{\circ} \mathrm{C} \pm 2^{\circ}\right)$ for 20 years in the LARMAUR lab at the University of Rennes with no light exposure until it was characterized. The second set included samples of two compositions that were drawn into fibers. The first composition was the Se-rich $\mathrm{GeSe}_{9}$ which lies well outside of the "reversibility window". The second composition was the archetypical $\mathrm{GeSe}_{4}$ stoichiometry which corresponds to the rigidity percolation threshold at $\langle r\rangle=2.4$ and lies within the "reversibility window". For this study, $\mathrm{GeSe}_{9}$ and $\mathrm{GeSe}_{4}$ fibers were fabricated at various times over the course of five years. The fibers were drawn in a He atmosphere at a speed of $1.22 \mathrm{~m} / \mathrm{min}$ and a tension of $20 \mathrm{~g}$. At this speed and tension, no measurable structural anisotropy could be observed by ${ }^{77} \mathrm{Se}$ Nuclear Magnetic Resonance, polarized Raman or X-ray Diffraction along the axis of the fibers. In these conditions, the quench rate was estimated at $\sim 500^{\circ} \mathrm{C} / \mathrm{min}^{3}$. After fabrication, the fibers were removed from their storage drums and stored in $15 \mathrm{~cm}$ long strands in silica tubes, in the dark at room temperature in the laboratory. Small pieces of the stored fibers were then removed from the silica tubes when DSC and Raman experiments were ready to be performed.

\section{B. Raman spectroscopy:}

Raman spectroscopy was performed on 20 year old bulk samples of each composition before and after rejuvenation in a DSC (See section C). Raman spectra were collected using a Renishaw InVia spectrometer with a 1200 lines/grating and a diode laser at a wavelength of $785 \mathrm{~nm}$ focused with a $10 \mathrm{X}$ objective at a power of $15 \mathrm{~mW}$ to avoid any photostructural changes. Spectra were collected for 120 seconds and averaged over three scans. Raman spectroscopy was also performed on small sections of $\mathrm{GeSe}_{9}$ and $\mathrm{GeSe}_{4}$ glass fibers from the same batch as that used for calorimetric measurements. Prior to each measurement, the sample surface was cleaned with alcohol and the fibers were lightly crushed and pressed into aluminum sample holders. Unpolarized Raman spectra were collected at ambient temperature and in backscattering geometry using a Bruker RFS 100/S Fourier-Transform Raman spectrometer equipped with a $\mathrm{Nd}$ :YAG laser operating at $1064 \mathrm{~nm}$ and a liquid nitrogen cooled solid-state Ge detector. A laser power level of $50 \mathrm{~mW}$ was used, and spectra were collected using a resolution of $2 \mathrm{~cm}^{-1}$. Approximately 100 scans were collected and averaged to obtain each Raman spectrum. The low photon energy and the low power level used to collect the Raman spectra, permitted to avoid any photostructural damage during data collection.

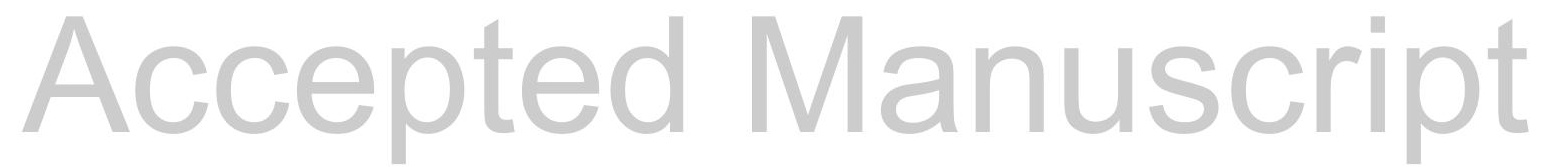




\section{Differential Scanning Calorimetry}

Enthalpy relaxation measurements were performed on 20 year old bulk samples of each composition using a TA Instrument DSC25 equipped with a refrigerated cooling system RCS90 and calibrated with an indium standard. Small bulk glass pieces were lightly polished to obtain a flat surface to optimize thermal contact with the DSC pan. Samples of mass $\sim 10 \mathrm{mg}$ were then hermetically sealed in a Tzero ${ }^{\circledR}$ aluminum pan. An identical empty pan was used as the reference. Samples were first heated at $10^{\circ} \mathrm{C} / \mathrm{min}$ up to $100^{\circ} \mathrm{C}$ above $T_{g}$ to measure the heat capacity of the relaxed glass. The sample was then rejuvenated by subjecting it to the same heattreatment as the original glass through equilibration at $T_{g}$ (up to $4 \mathrm{~h}$ ) and slowly cooling at $1^{\circ} \mathrm{C} / \mathrm{min}$ to room temperature ${ }^{40}$. A run of the rejuvenated glass was then immediately collected at $10^{\circ} \mathrm{C} / \mathrm{min}$ over the same temperature range. The two thermograms were integrated and subtracted to calculate the relaxed enthalpy of the 20 year old sample. Enthalpy relaxation measurements were also performed on glass fibers using a Mettler Toledo DSC1 calorimeter equipped with a FRS5 sensor and sub-ambient cooling unit. DSC was performed on both sets of $\mathrm{GeSe}_{9}$ and $\mathrm{GeSe}_{4}$ fibers of varying ages, in standard (non-modulated) mode. Prior to any experiments, both the temperatures and the heat flow were calibrated using indium and zinc standards. Heat capacity values were calculated from the heat flow using the molar mass of the samples. Tested samples consisted of $3 \mathrm{~mm}$ long pieces of fiber, placed side by side in the center of an aluminum DSC pan and hermetically sealed. Empty aluminum pans were used as a reference, and sample masses were in the range of 5 to $7 \mathrm{mg}$. Enthalpy change calculations were completed using two consecutive heating scans of each sample. To perform such a calculation, each fiber sample was placed in the DSC and heated at $10 \mathrm{C} \mathrm{min}^{-1}$ to a temperature well above $T_{g}$, allowed to equilibrate at this temperature, and subsequently cooled in the DSC using the same $10 \mathrm{C} \mathrm{min}^{-1}$ rate to well below $T_{g}$. This first temperature up-scan captures the enthalpy of the fiber due to relaxation. Upon completion of cooling, the sample is then reheated, again at $10 \mathrm{C}$ $\min ^{-1}$, to obtain the reference DSC curve. Enthalpy change is then determined by the integration of the area between the two heat flow curves.

\section{RESULTS}

\section{A. 20 year old bulk samples:}

\section{Calorimetry}

The DSC curves of all Ge-Se bulk glasses after 20 year aging at room temperature are shown in Fig. 2. Each curve is compared with that of the same glass after rejuvenation. Glasses with lower $T_{g}$ exhibit large endothermic peaks corresponding to the regain of relaxed enthalpy during reheating. The large relaxed enthalpy is consistent with the shorter relaxation time associated with the lower $T_{g}$ since the $A$ term in the TNM equation (Eq. 1) is exponentially dependent on $T_{g}$. Moreover, the large relaxed enthalpy $(>300 \mathrm{~J} / \mathrm{mol})$ is also consistent with the higher fragility $m$ 
of these glasses ${ }^{35}$. As shown in Fig. 1b, higher fragility leads to steeper departure from equilibrium and greater build up for relaxation. Interestingly, glasses within the so-called "reversibility window" also show rather large relaxed enthalpy (up to $167.5 \mathrm{~J} / \mathrm{mol}$ ) despite their higher $T_{g}$ and the reduced thermodynamic potential for relaxation associated with their lower fragility. Finally, glasses with $T_{g}$ values far above room temperature exhibit small but still measurable enthalpy relaxation as shown for $\mathrm{Ge}_{30} \mathrm{Se}_{70}$. Overall, these data clearly indicate that all glasses, including those in the "reversibility window" can undergo significant structural relaxation. As shown in the inset of Fig. 2, the ability to relax at room temperature is directly dependent on the $T_{g}$ of the glass and therefore appears to be mainly limited by kinetic factors.

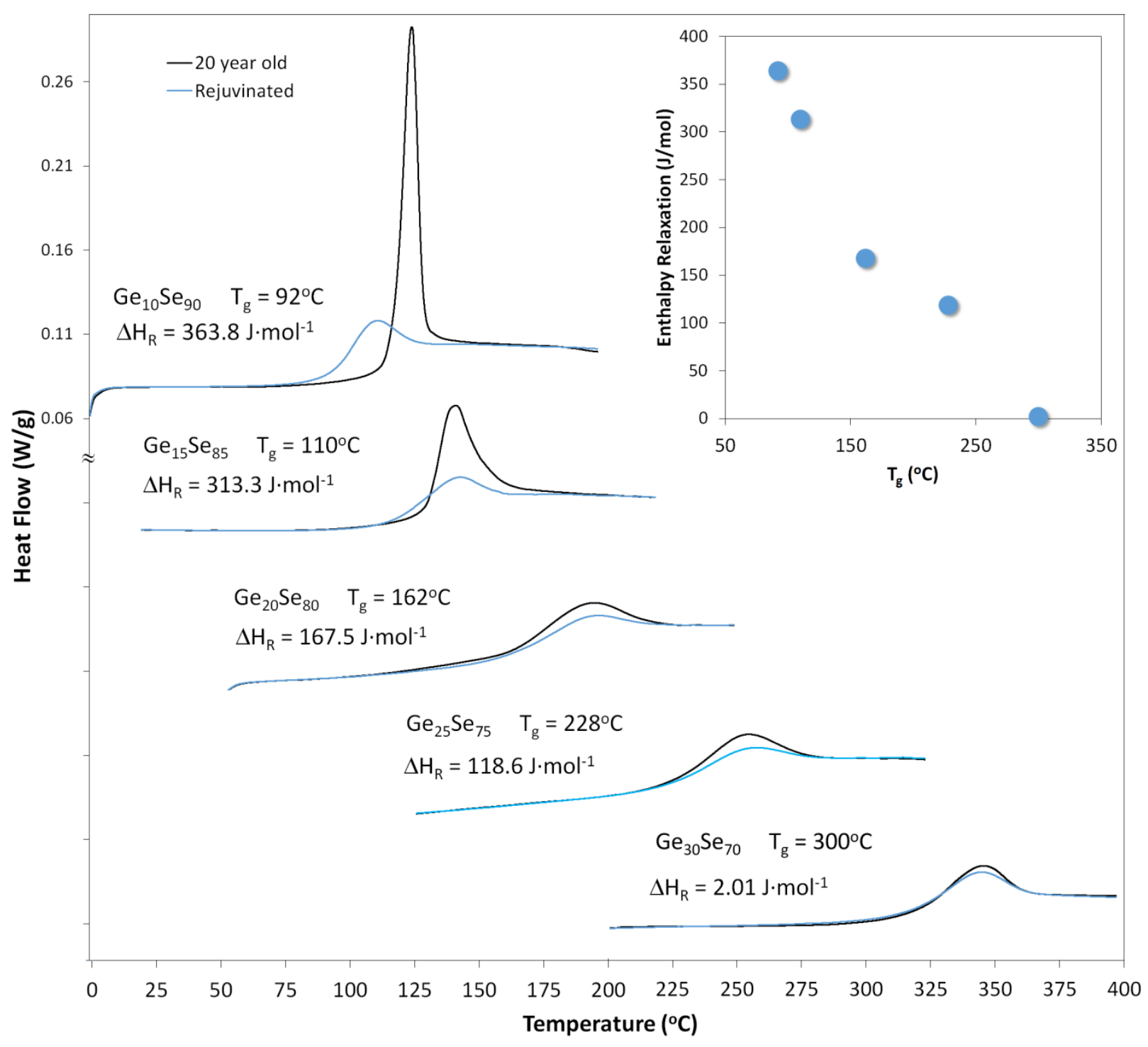


Fig. 2: DSC curves of bulk Ge-Se glasses after 20 year annealing at room temperature $(295 \mathrm{~K})$. The relaxed enthalpy $\Delta \mathrm{H}_{\mathrm{R}}$ corresponds to the difference between the integrated curves of the relaxed and rejuvenated glass. The inset shows the dependence of $\Delta \mathrm{H}_{\mathrm{R}}$ on the glass transition temperature $T_{g}$. (endothermic is up)

\section{Raman spectroscopy}

As shown in Fig. 3, the Raman spectra of all Ge-Se glasses exhibit the characteristic three main spectral lines located at $255 \mathrm{~cm}^{-1}, 213 \mathrm{~cm}^{-1}$ and $195 \mathrm{~cm}^{-1}$ which corresponds to the Se-chain modes and the edge-sharing and corner-sharing tetrahedral modes, respectively ${ }^{5,43}$. The spectra normalized to the Se-chain mode show a systematic decrease in edge-sharing tetrahedra modes with annealing while the corner-sharing mode concomitantly increases. This indicates that a conversion from edge- to corner-sharing tetrahedra is a structural marker of aging in these glasses. In order to further investigate a correlation between this structural evolution and enthalpy relaxation, the aging process was monitored over time in a series of Ge-Se glass fibers as detailed in the following section.
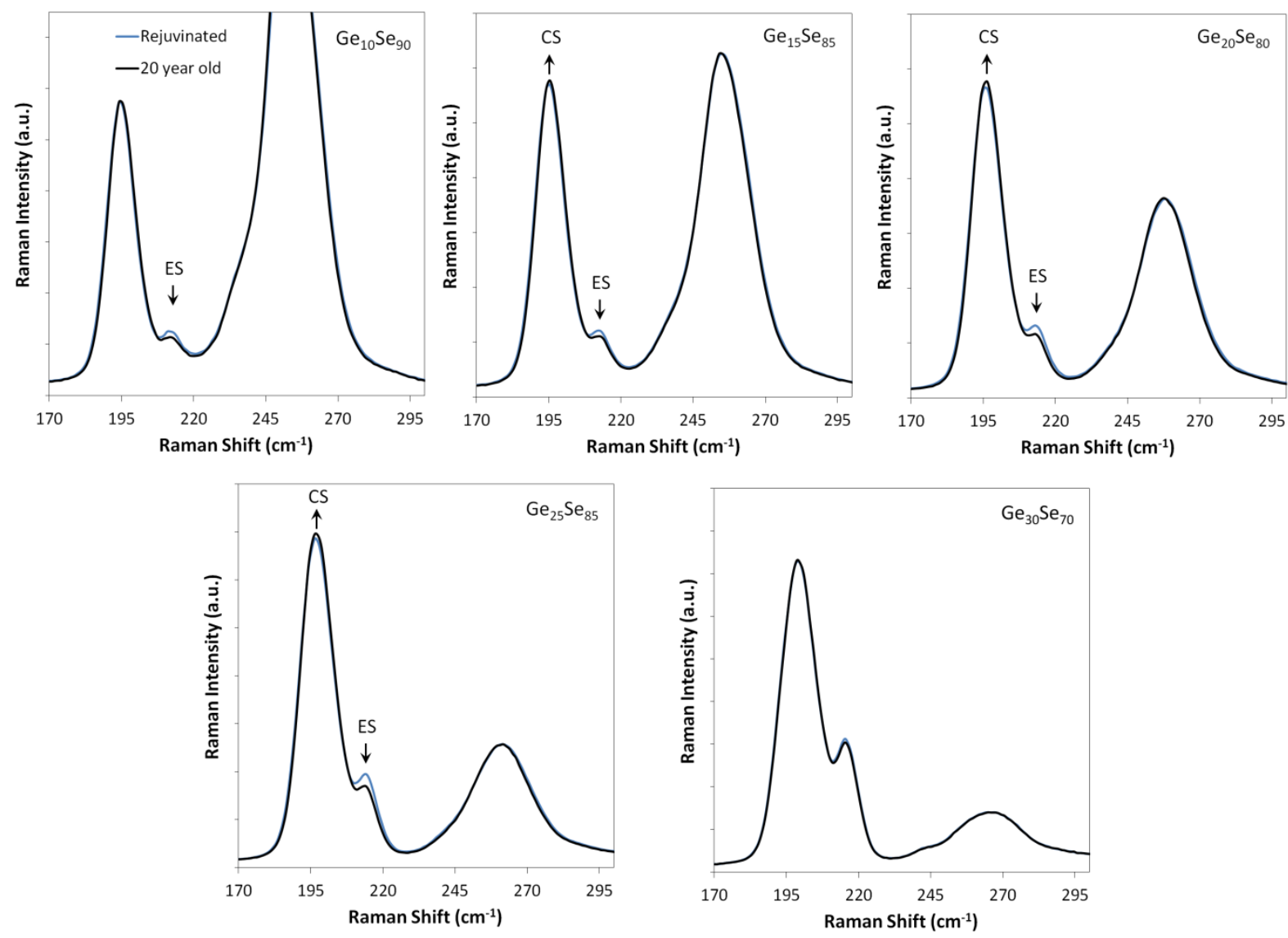
Fig. 3: Raman spectra of bulk Ge-Se glasses after 20 year aging at room temperature (295 K) compared with the rejuvenated glass. The peak intensity ratio between corner- and edge-sharing tetrahedra systematically increases with annealing.

\section{B. 5 year old fiber samples}

\section{Calorimetry}

Figure 4 presents the heat capacity curves of $\mathrm{GeSe}_{9}$ and $\mathrm{GeSe}_{4}$ glass fibers collected at several time intervals as they were aging at room temperature for up to five years. The DSC curves reveal notably different evolutions between the two fibers which correspond to the expected behavior of glasses annealed at different temperatures relative to $T_{g}$ (for $\mathrm{GeSe}_{9}: T_{g}-T_{a} \sim 70^{\circ} \mathrm{C}$ while for $\mathrm{GeSe}_{4}: T_{g}-T_{a} \sim 140^{\circ} \mathrm{C}$ ). The $\mathrm{GeSe}_{9}$ shows a large $C_{p}$ overshoot while the $\mathrm{GeSe}_{4}$ shows the development of a pre- $T_{g}$ endotherm that slowly grows with aging. The main DSC features of these two glasses can be predictably reproduced using the TNM modeling procedure of Hodge ${ }^{44}$ as shown in Fig. 5. The $\mathrm{GeSe}_{9}$ and $\mathrm{GeSe}_{4}$ glasses were modeled using a cooling rate of $500^{\circ} \mathrm{C} / \mathrm{min}^{3}$ and a reheating rate of $10^{\circ} \mathrm{C} / \mathrm{min}$ after annealing at $25^{\circ} \mathrm{C}$ (room temperature) for increasing time periods. Modeling parameters were estimated from literature for fragility index $m^{35}$, non-exponentiality $\beta^{45}$ and non-linearity $x^{46}$. The modeled and experimental $C_{p}$ curves show good qualitative match. Figure $4 \mathrm{a} \& 5$ a shows that, as a result of its low $T_{g}$, the $\mathrm{GeSe}_{9}$ glass can relax rapidly at room temperature and exhibits a large overshoot of $C_{p}$ after only 3.5 years. This overshoot is indicative of a large relaxed enthalpy enhanced by its higher fragility, as predicted from Fig. 1. In contrast, the $C_{p}$ of the $\mathrm{GeSe}_{4}$ glass in Fig. $4 \mathrm{~b} \& 5 \mathrm{~b}$ shows the development of a pre- $T_{g}$ endotherm that shifts to higher temperatures upon longer annealing. This endotherm is the well-known signature of fast relaxing domains regaining enthalpy upon reheating after a long anneal. Such behaviors have been described in great detail by Hodge both

experimentally and theoretically ${ }^{44,47-51}$. Such pre- $T_{g}$ endotherms are only observable for a specific range of annealing temperatures far below $T_{g}$ after a fast quench ${ }^{44,47}$, which is precisely the case for the $\mathrm{GeSe}_{4}$ fibers. For glasses with $T_{g}$ closer to their annealing temperature like $\mathrm{GeSe}_{9}$, the entire distribution of relaxation times is sufficiently short that relaxation occurs quickly at room temperature, and upon reheating the enthalpy relaxation appears as the conventional overshoot superimposed on the $T_{g}$, as shown in Fig. 4a \& 5a. Overall, both glasses show the characteristic relaxation behavior predicted by the TNM model.

\section{Raman spectroscopy}

Figure 6 shows the spectra of the $\mathrm{GeSe}_{4}$ glass fibers collected by FT-Raman. The spectra show the characteristic Se-chain and tetrahedral modes near $250 \mathrm{~cm}^{-1}$ and $200 \mathrm{~cm}^{-1}$ respectively as well as the low frequency broadband Se bending modes near $100 \mathrm{~cm}^{-1} 43$ (Fig. 6a). Upon close inspection of the tetrahedral modes, it is found that the relative intensity of the edge-sharing mode progressively decreases as a function of aging, as depicted in Fig. 6b. This change is 
observed in both glasses. A similar evolution of edge-sharing mode intensity as a function of temperature has been previously observed in the supercooled liquid of $\mathrm{GeSe}_{4}{ }^{43}$. The edgesharing mode was shown to decrease with decreasing temperature of the liquid. In the present case the edge-sharing mode intensity is found to decrease with decreasing fictive temperature of the glass as it relaxes towards equilibrium upon aging. This indicates that the structural evolution observed by Raman is related to changes in thermal properties (enthalpy) of the glass. Indeed, a strong correlation between the structural and thermal properties can be revealed by plotting the time evolution of the enthalpy and Raman features as shown in Fig. 7 for $\mathrm{GeSe}_{4}$. In Fig. 7(a) the ratio of the intensities of the edge- and the corner- sharing tetrahedral modes is normalized and plotted as a function of aging time. The decay of the spectral ratio is found to follow a stretched exponential function (Kohlrausch function). In Fig. 7(b), the relaxed enthalpy calculated from data in Fig. 4(b) is similarly normalized and plotted as function of time over the same period. It is found that the enthalpy decay can be fitted with the same stretched exponential function with a relaxation time $\tau=294$ days and a stretching exponent $\beta=0.686$. This result confirms that the two relaxation processes are strongly correlated.

\section{Accepted Manuscript}



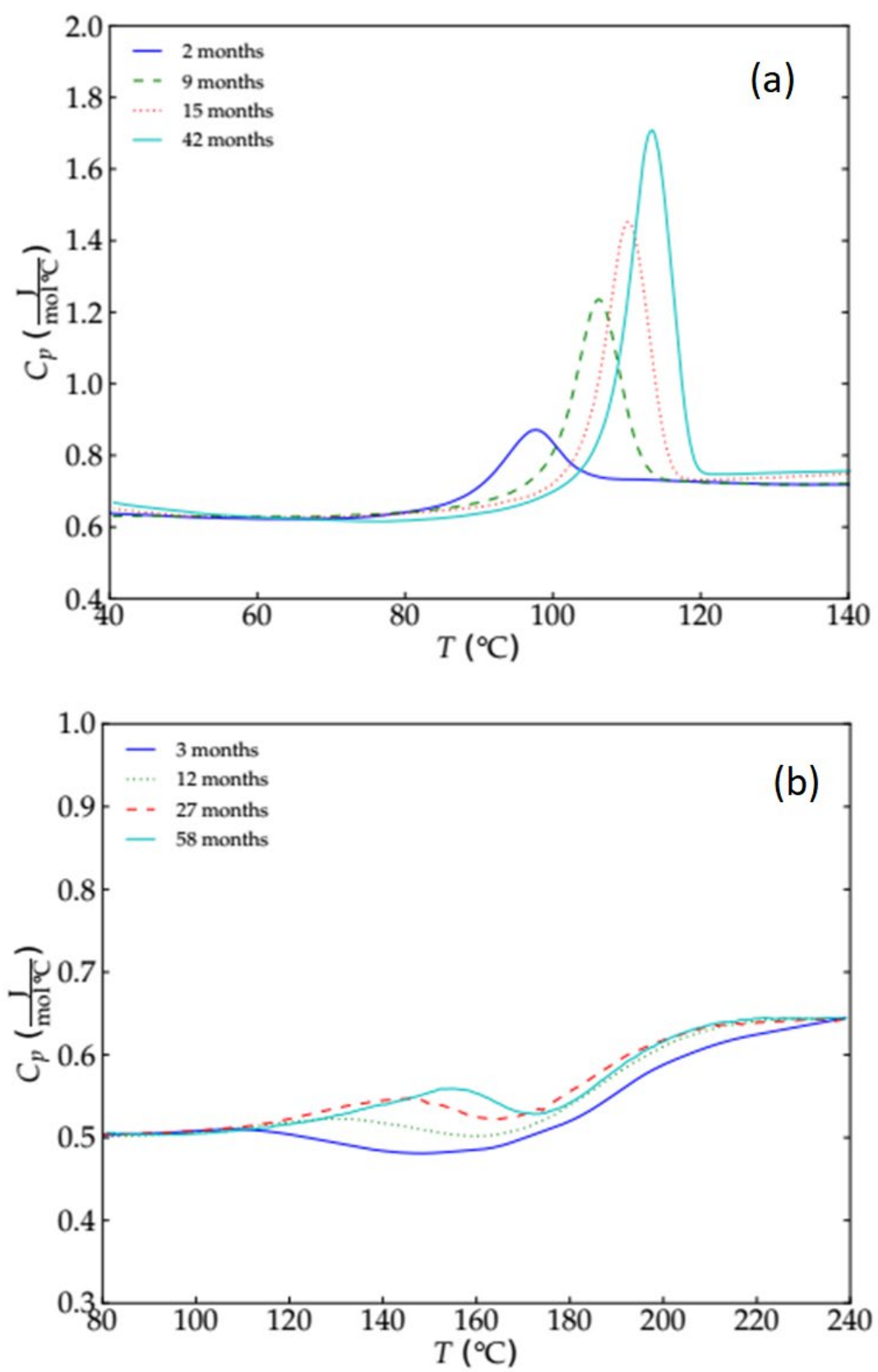

Fig. 4: Experimental heat capacity curves for (a) $\mathrm{GeSe}_{9}$ glass fibers annealed at room temperature for up to 3.5 years and (b) $\mathrm{GeSe}_{4}$ glass fibers annealed at room temperature for up to 5 years. 

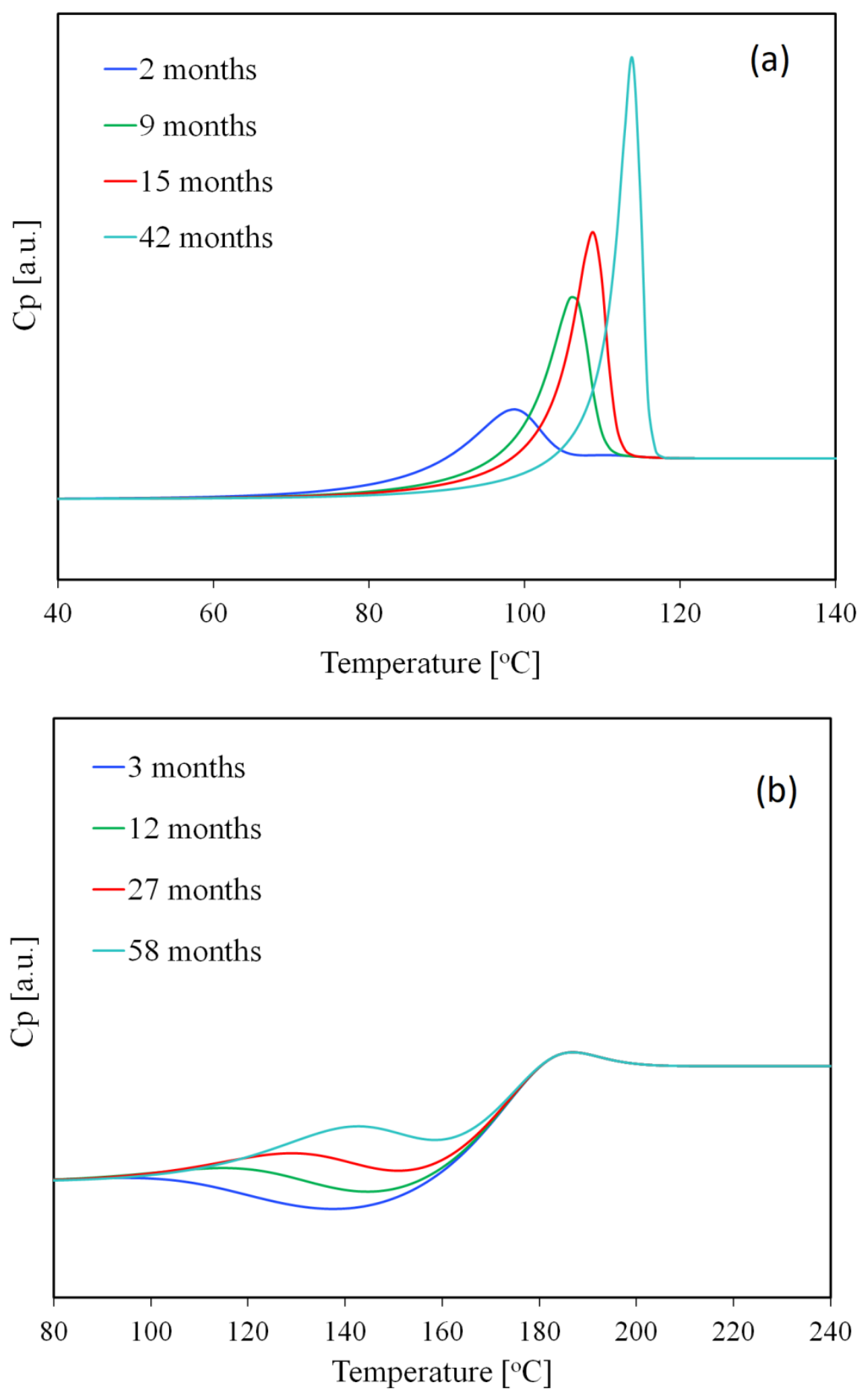

Fig. 5: Calculated heat capacity curves as a function of annealing at room temperature following the TNM modeling procedure developed by Hodge ${ }^{44}$ for (a) $\mathrm{GeSe}_{9}$ glass using model parameters $\beta=0.54 ; x=$ $0.51 ; m=38$ and $T_{g}=365 \mathrm{~K}$; (b) $\mathrm{GeSe}_{4}$ glass using model parameters $\beta=0.60 ; x=0.57 ; m=27$ and $T_{g}$ $=435 \mathrm{~K}$. 

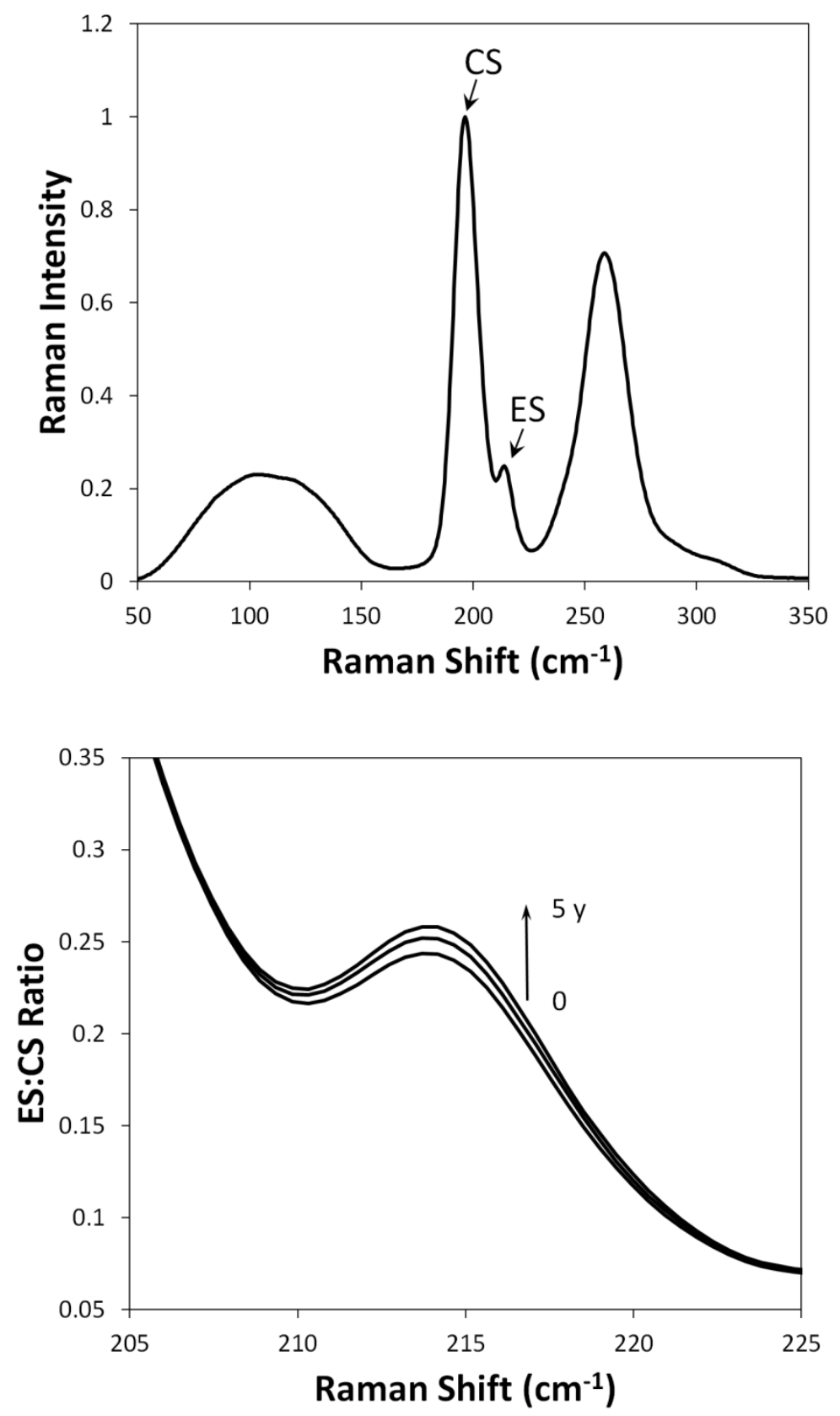

Fig. 6: (a) Typical unpolarized Raman spectrum of $\mathrm{GeSe}_{4}$ glass showing the two modes associated with corner-sharing and edge-sharing tetrahedra at $195 \mathrm{~cm}^{-1}$ and $213 \mathrm{~cm}^{-1}$ respectively; (b) Increase of the relative intensity of the edge-sharing mode upon aging at room temperature for 0,2 and 5 years (all spectra are normalized to the intensity of the corner-sharing mode).

The same construction can be performed for the $\mathrm{GeSe}_{9}$ glass as shown in Fig. 8. Again, Raman features and enthalpy both follow a stretched exponential decay that can be fitted with the same function. In the case of $\mathrm{GeSe}_{9}$ the relaxation time is $\tau=118$ days and the stretching exponent 
factor is $\beta=0.424$. As expected, the lower $T_{g}$ glass has a shorter relaxation time at room temperature consistent with results of Fig. 4(a). The stretching exponent values also show that the non-exponential factor $\beta$ is notably smaller for the fragile glass $\left(\mathrm{GeSe}_{9} m \sim 38\right)$ than the strong glass $\left(\mathrm{GeSe}_{4} m \sim 27\right)$. The two glasses are likely still far from equilibrium, hence, these values of $\beta$ are only estimates, nevertheless they are consistent with expected trend between fragility and non-exponentiality, and the $\beta$ values obtained in this study are in good agreement with previously reported data ${ }^{45}$.
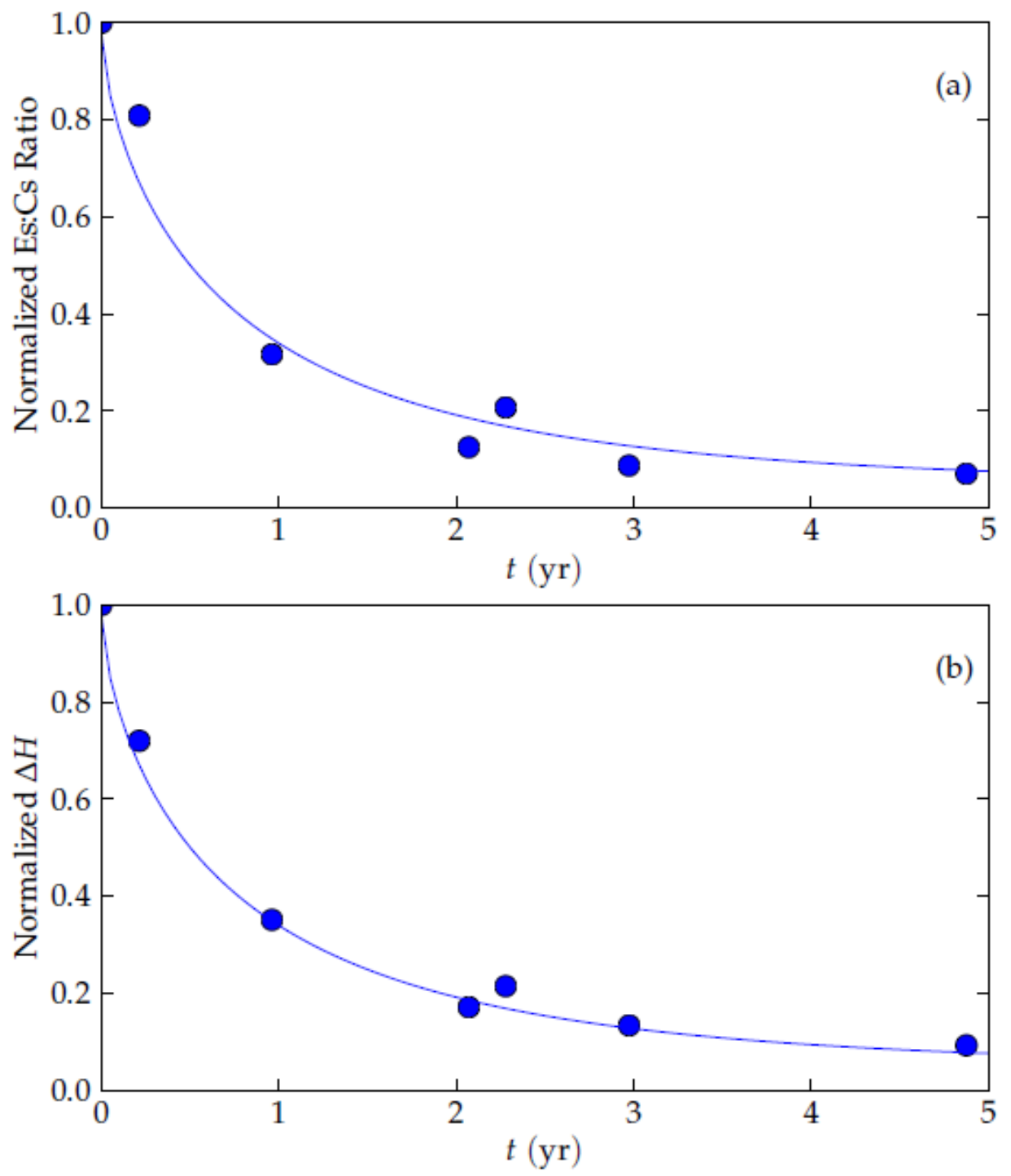

Fig. 7: (a) Normalized ratio of the intensities of the edge- and the corner-sharing tetrahedral Raman modes of $\mathrm{GeSe}_{4}$ glass as a function of aging time at room temperature; (b) Normalized enthalpy relaxation of $\mathrm{GeSe}_{4}$ glass as a function of aging time at room temperature. The line in both graphs correspond to the same stretched exponential function with $\tau=294$ days and $\beta=0.686$ 

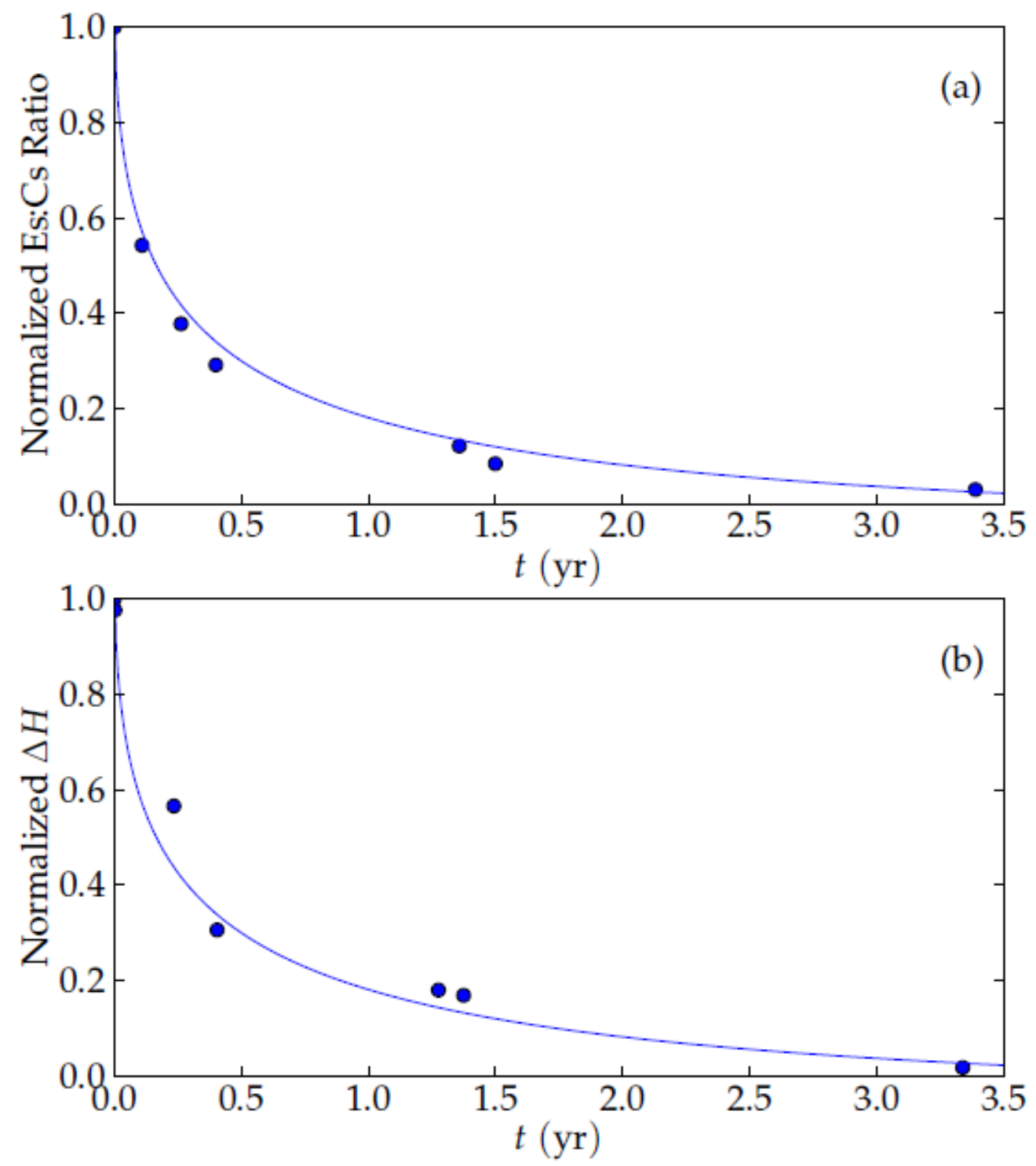

Fig. 8: (a) Normalized ratio of the intensities of the edge- and the corner-sharing tetrahedral Raman modes of $\mathrm{GeSe}_{9}$ glass as a function of aging time at room temperature; (b) Normalized enthalpy relaxation of $\mathrm{GeSe}_{9}$ glass as a function of aging time at room temperature. The line in both graphs correspond to the same stretched exponential function with $\tau=118$ days and $\beta=0.424$

\section{DISCUSSION}

\section{A. Conventional relaxation behavior in the "reversibility window"}

Overall, the results of Fig. $2 \& 5$ reveal that given enough time, all Ge-Se glasses undergo a sizeable enthalpy relaxation even at room temperature. The relative extent of relaxation of all glasses is consistent with expectations for samples annealed at different temperatures relative to 
$T_{g}$. Hence, it can be concluded that glasses from the so-called "reversibility window" do not exhibit any unusual resistance to relaxation, granted sufficient observation time is allowed for the measurement. The features of the DSC curves and their evolution with aging are also well predicted by the TNM model for glasses inside and outside the so-called "reversibility window". The multiple features of the DSC curves are fully consistent with their relative fragility and $T_{g}$ values. Furthermore, the time dependence of the enthalpy relaxation for both glasses exhibit a stretched exponential decay characteristic of conventional amorphous systems during sub- $T_{g}$ relaxation ${ }^{24}$. The non-exponential factors $\beta$ describing the decay are also found to be in good agreement with expectations from glasses with their relative fragilities ${ }^{45}$. Hence, all Ge-Se glasses exhibit a rather conventional relaxation behavior and no abnormal relaxation properties are observed for glasses within the "reversibility window", in fact these glasses behave exactly as expected from model predictions.

\section{B. Structural origin of relaxation in Ge-Se glasses.}

As shown in Fig. $3 \&$, aging of Ge-Se glasses is systematically associated with a conversion of edge-sharing tetrahedra to corner-sharing tetrahedra. The magnitude and kinetics of this structural change is closely related to enthalpy relaxation for each composition studied. This behavior can be explained in two ways, based on considerations of density and configurational entropy. In terms of density, it is expected that the reduced structural dimensionality imparted by edge-sharing tetrahedra leads to poorer packing efficiency and in turn to lower volumetric density. For example, the corner-sharing 3D crystalline polymorph of $\mathrm{GeSe}_{2}$ is $9.6 \%$ denser than the corner- and edge-sharing $2 \mathrm{D}$ polymorph consisting of layers ${ }^{52}$. This trend is also broadly observed in tetrahedral liquids such as $\mathrm{GeSe}_{4}$ and $\mathrm{ZnSe}_{2}$ where the fraction of edge-sharing units decreases with lower temperature and higher density ${ }^{43,53,54}$. In the case of Ge-Se glasses, the decrease in edge-sharing units is therefore consistent with the volume contraction associated with lowering of the fictive temperature during aging. In terms of configurational entropy, the relaxation process is associated with a decrease in entropy towards the equilibrium liquid line as illustrated schematically in Fig. 1(b). This entropy loss upon aging can indeed be observed experimentally in the heat capacity curves of Fig. $2 \& 4$ where the $C_{p}$ overshoot is representative of the regain in lost entropy upon reheating. This decrease in entropy can also be qualitatively

linked to the structural changes observed by Raman using the constraint counting formalism ${ }^{55-57}$. As previously described ${ }^{54}$, a pair of edge-sharing tetrahedra possess two fewer angular constraints than a pair of corner-sharing tetrahedra. Hence, a decrease in edge-sharing tetrahedra will lead to a decrease in network degrees of freedom $f$ and in turn to a decrease in configurational entropy $S_{c}$ according to:

$$
S_{C}=f N k \ln \Omega
$$

where $\Omega$ is the number of accessible microstates per atomic degree of freedom (independent of $f$ ) 55,56. The structural mechanism for entropy relaxation therefore appears to be related to the backbone of tetrahedral units in the glassy structure. Interestingly, that mechanism is identical in 
both glasses, as shown in Figs. $7 \&$ 8. This further confirms that glasses from the "reversibility window" do not exhibit any abnormal relaxation behavior relative to other compositions from the same system.

\section{Non-reversing enthalpy and MDSC}

The results described above conclusively indicate that glasses within the "reversibility window" can undergo significant enthalpy relaxation. This raises questions regarding the validity of the non-reversing enthalpy $\Delta H_{n r}$ as a means of assessing the relaxation behavior of glasses. Indeed it has been broadly suggested that vanishing values of $\Delta H_{n r}$ are indicative of a resistance to aging 9,15-17. However, the present results clearly indicate that this is not the case. The physical interpretation of $\Delta H_{n r}$ therefore remains unclear and in fact it has been highly controversial since the early development of MDSC. In particular, Schawe ${ }^{58}$ emphasizes that $\Delta H_{n r}$ is the difference between two quantities that are measured with two different time constants: the underlying heat flow associated with the time constant of the average heating rate and the reversible heat flow associated with the time constant of the superimposed oscillation. Schawe then concludes that "the non-reversing component is determined by subtracting two logically different quantities that are not directly comparable" ${ }^{58}$. Nevertheless, $\Delta H_{n r}$ has been tentatively equated to the enthalpy relaxation upon annealing ${ }^{59}$, but Hutchinson et al. ${ }^{19}$ showed experimentally that this equality does not actually hold. Instead, MDSC signals can be effectively interpreted in terms of complex, in-phase and out-of-phase heat capacities with a real part $C^{\prime}$ (storage heat capacity) and an imaginary part $C$ " (loss heat capacity) ${ }^{33,60-62}$. It should be noted that the use of the complex heat capacity is contingent upon a linear response between the heating rate and heat flow which should be ascertained through construction of a Lissajous plot ${ }^{33,63}$. When correctly implemented, this approach permits to extract the fragility by measuring the shift of the loss peak with oscillating frequency ${ }^{62}$ and can also provide estimates of the stretching exponent $\beta^{64}$. These two parameters provide a more complete and reliable assessment of the relaxation properties of a glass-forming system than $\Delta H_{n r}$.

\section{CONCLUSIONS}

In this study, Ge-Se glasses lying outside and inside the "reversibility window" were annealed at room temperature for up to twenty years. The purpose of this experiment was to test whether glasses from the "reversibility window" exhibit any resistance to physical aging as previously claimed. A sizable enthalpy relaxation was observed for all glasses including those within the socalled "reversibility window". Furthermore, the relaxation process obeys a stretched exponential decay characteristic of amorphous systems. The heat capacity curves of each glass followed the behavior expected from the TNM model for systems annealed far from $T_{g}$. The values of stretching exponent $\beta$ and relaxation time $\tau$ were also consistent with expectations. These results demonstrate that glasses from the "reversibility window" do not show any particular resistance to relaxation or aging. In addition, Raman spectra of both glasses reveal that structural relaxation is associated with a conversion of edge-sharing to corner-sharing tetrahedra. This structural 
evolution is consistent with an increase in density and a decrease in configurational entropy. The structural mechanism is identical in both glasses and further confirms that glasses from the "reversibility window" do not show any unusual relaxation behavior relative to other compositions in the same system. Finally, these results invalidate the interpretation of a vanishing value of $\Delta H_{n r}$ as indicative of a resistance to aging. This is an important consideration in view of the increasing popularity of MDSC and its extension to the characterization of high temperature oxide glasses ${ }^{63}$.

\section{ACKNOWLEDGEMENT}

PL acknowledge financial support from NSF-DMR under grant\#: 1832817. We thank Isabelle Melscoët for synthesizing the bulk Ge-Se glasses.

\section{DATA AIVAILABILITY}

The data that support the findings of this study are available from the corresponding author upon reasonable request.

\section{REFERENCES}

${ }^{1}$ R. C. Welch, J. R. Smith, M. Potuzak, X. Guo, B. F. Bowden, T. J. Kiczenski, D. C. Allan, E. A. King, A. J. Ellison, and J. C. Mauro, "Dynamics of Glass Relaxation at Room Temperature" Physical Review Letters 110, 265901 (2013)

${ }^{2}$ R. P. Wang, A. V. Rode, S. J. Madden, C. J. Zha, R. A. Jarvis, and B. Luther-Davies, "Structural relaxation and optical properties in amorphous Ge33As12Se55 films" J. Non-Cryst. Solids 353, 950 (2007)

${ }^{3}$ P. Lucas, E. A. King, Y. Gueguen, J.-C. Sangleboeuf, V. Keryvin, R. G. Erdmann, G. Delaizir, C. Boussard-Pledel, B. Bureau, X.-H. Zhang, and T. Rouxel, "Correlation between thermal and mechanical relaxation in chalcogenide glass fibers" J. Am. Ceram. Soc. 92, 1986 (2009)

${ }^{4}$ R. Golovchak, H. Jain, O. Shpotyuk, A. Kozdras, A. Saiter, and J. M. Saiter, "Experimental verification of the reversibility window concept in binary As-Se glasses subjected to a long-term physical aging" Phys. Rev. B: Condens. Matter 78, 014202/1 (2008)

${ }^{5}$ P. Lucas, E. A. King, O. Gulbiten, J. L. Yarger, E. Soignard, and B. Bureau, "Bimodal phase percolation model for the structure of Ge-Se glasses and the existence of the intermediate phase" Phys. Rev. B: Condens. Matter 80, 214114/1 (2009)

${ }^{6}$ E. L. Gjersing, S. Sen, and R. E. Youngman, "Mechanistic understanding of the effect of rigidity percolation on structural relaxation in supercooled germanium selenide liquids" Phys. Rev. B: Condens. Matter Mater. Phys. 82, 014203/1 (2010)

${ }^{7}$ S. Sen, D. C. Kaseman, I. Hung, and Z. Gan, "77Se Nuclear Spin-Lattice Relaxation in Binary Ge-Se Glasses: Insights into Floppy Versus Rigid Behavior of Structural Units" J. Phys. Chem. B 119, 5747 (2015)

${ }^{8}$ J. C. Phillips, "Topology of covalent non-crystalline solids. I. Short-range order in chalcogenide alloys" J. Non-Cryst. Solids 34, 153 (1979)

\section{Accepted Manuscript}


${ }^{9}$ S. Bhosle, K. Gunasekera, P. Chen, P. Boolchand, M. Micoulaut, and C. Massobrio, "Meeting experimental challenges to physics of network glasses: Assessing the role of sample homogeneity" Solid State Commun. 151, 1851 (2011)

${ }^{10}$ M. F. Thorpe, "Continuous deformations in random networks" J. Non-Cryst. Solids 57, 355 (1983)

${ }^{11}$ H. He, and M. F. Thorpe, "Elastic properties of glasses" Phys. Rev. Lett. 54, 2107 (1985)

${ }^{12}$ U. Senapati, K. Firstenberg, and A. K. Varshneya, "Structure-property inter-relations in chalcogenide glasses and their practical implications" J. Non-Cryst. Solids 222, 153 (1997)

${ }^{13}$ R. P. Wang, A. Smith, B. Luther-Davies, H. Kokkonen, and I. Jackson, "Observation of two elastic thresholds in GexAsySe1-x-y glasses" J. Appl. Phys. 105, 056109/1 (2009)

${ }^{14}$ M. F. Thorpe, D. J. Jacobs, M. V. Chubynsky, and J. C. Phillips, "Self-organization in network glasses" J. Non-Cryst. Solids 266-269, 859 (2000)

${ }^{15}$ S. Chakravarty, D. G. Georgiev, P. Boolchand, and M. Micoulaut, "Ageing, fragility and the reversibility window in bulk alloy glasses" J. Phys.: Condens. Matter 17, L1 (2005)

${ }^{16}$ P. Boolchand, G. Lucovsky, J. C. Phillips, and M. F. Thorpe, "Self-organization and the physics of glassy networks" Philosophical Magazine 85, 3823 (2005)

${ }^{17}$ U. Vempati, and P. Boolchand, "The thermally reversing window in ternary GexPxS1-2x glasses" J. Phys.: Condens. Matter 16, S5121 (2004)

${ }^{18}$ E. Verdonck, K. Schaap, and L. C. Thomas, "A discussion of the principles and applications of Modulated Temperature DSC (MTDSC)" International Journal of Pharmaceutics 192, 3 (1999)

${ }^{19}$ J. M. Hutchinson, A. B. Tong, and Z. Jiang, "Aging of polycarbonate studied by temperature modulated differential scanning calorimetry" Thermochim. Acta 335, 27 (1999)

${ }^{20}$ M. A. DeBolt, A. J. Easteal, P. B. Macedo, and C. T. Moynihan, "Analysis of structural relaxation in glass using rate heating data" J. Am. Ceram. Soc. 59, 16 (1976)

${ }^{21}$ I. M. Hodge, and A. R. Berens, "Calculation of the effects of annealing on Tg endotherms"

Macromolecules 14, 1598 (1981)

${ }^{22}$ G. W. Scherer, "Volume Relaxation Far from Equilibrium" J. Am. Ceram. Soc. 69, 374 (1986)

${ }^{23}$ H. L. Ma, X. H. Zhang, J. Lucas, and C. T. Moynihan, "Relaxation near room temperature in tellurium chalcohalide glasses" J. Non-Cryst. Solids 140, 209 (1992)

${ }^{24}$ G. W. Scherer, "Theory of relaxation" J. Non-Cryst. Solids 123, 75 (1990)

${ }^{25}$ I. M. Hodge, "Physical aging in polymer glasses" Science (Washington, D. C.) 267, 1945 (1995)

${ }^{26}$ H. S. Chen, and C. R. Kurkjian, "Sub-Sub-T ${ }_{g}$ Enthalpy Relaxation in a $\mathrm{B}_{2} \mathrm{O}_{3}$ Glass" J. Am. Cer. Soc. 66, $613(1983)$

${ }^{27}$ J.S. Sanghera, J.D. Mackenzie, and S.-Y. Ryou, "Shrinkage of fluorozirconate glass fibers at low temperatures" J. Non-Cryst. Solids 125, 76 (1990)

${ }^{28}$ C. T. Moynihan, S. M. Opalka, R. Mossadegh, S. N. Crichton, and A. J. Bruce, "Structural relaxation in fluoride glasses" NATO ASI Ser., Ser. E 123, 163 (1987)

${ }^{29}$ M. Ya, J. Deubener, and Y. Yue, "Enthalpy and Anisotropy Relaxation of Glass Fibers" J. Am. Ceram. Soc. 91, 745 (2008)

${ }^{30} \mathrm{~L}$. Hu, and Y. Yue, "Secondary Relaxation in Metallic Glass Formers: Its Correlation with the Genuine Johari-Goldstein Relaxation" J. Phys. Chem. C 113, 15001 (2009)

${ }^{31}$ H. B. Yu, M. Tylinski, A. Guiseppi-Elie, M. D. Ediger, and R. Richert, "Suppression of beta Relaxation in Vapor-Deposited Ultrastable Glasses" Physical Review Letters 115, 185501 (2015)

${ }^{32}$ Q. Zheng, and J. C. Mauro, "Variability in the relaxation behavior of glass: Impact of thermal history fluctuations and fragility" The Journal of Chemical Physics 146, 074504 (2017)

${ }^{33}$ O. Gulbiten, J. C. Mauro, and P. Lucas, "Relaxation of enthalpy fluctuations during sub-Tg annealing of glassy selenium" J. Chem. Phys. 138, 244504/1 (2013)

${ }^{34}$ X. Guo, J. C. Mauro, D. C. Allan, and M. M. Smedskjaer, "Predictive model for the composition dependence of glassy dynamics" Journal of the American Ceramic Society 101, 1169 (2017)

${ }^{35}$ A. Zeidler, P. S. Salmon, D. A. J. Whittaker, K. J. Pizzey, and A. C. Hannon, "Topological Ordering and Viscosity in the Glass-Forming Ge-Se System: The Search for a Structural or Dynamical Signature of the Intermediate Phase" Frontiers in Materials 4, (2017)

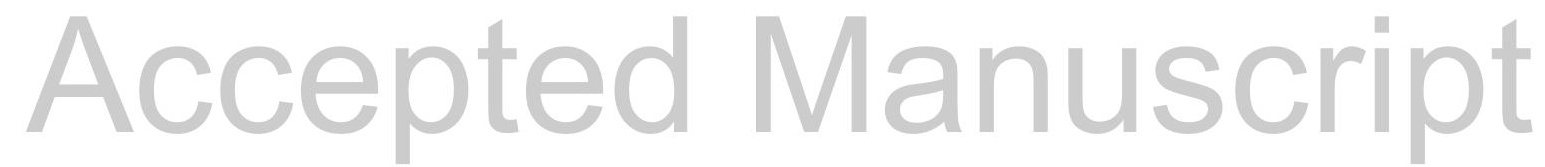


${ }^{36}$ L. M. Martinez, and C. A. Angell, "A thermodynamic connection to the fragility of glass-forming liquids" Nature (London, U. K.) 410, 663 (2001)

${ }^{37}$ W. Kauzmann, "The Nature of the Glassy State and the Behavior of Liquids at Low Temperatures" Chemical Reviews 43, 219 (1948)

${ }^{38}$ L.-M. Wang, V. Velikov, and C. A. Angell, "Direct determination of kinetic fragility indices of glassforming liquids by differential scanning calorimetry: kinetic versus thermodynamic fragilities" J. Chem. Phys. 117, 10184 (2002)

${ }^{39}$ C. T. Moynihan, S. K. Lee, M. Tatsumisago, and T. Minami, "Estimation of activation energies for structural relaxation and viscous flow from DTA and DSC experiments" Thermochim. Acta 280/281, 153 (1996)

${ }^{40}$ I. Melscoet, "Synthese, Characterization et Optimization de Verre Optiques pour des Application Infrarouge dans la fenetre 8-12 micorns" PhD Thesis, University of Rennes, Rennes France, (Dec 2000)

${ }^{41}$ P. Lucas, G. J. Coleman, S. Sen, S. Cui, Y. Guimond, L. Calvez, C. Boussard-Pledel, B. Bureau, and J. Troles, "Structural and chemical homogeneity of chalcogenide glass prepared by melt-rocking" J. Chem. Phys. 150, 014505/1 (2019)

${ }^{42}$ J. P Guin, T. Rouxel, J. C. Sangleboeuf, I. Melscoet, and J. Lucas, "Hardness, toughness, and scratchability of germanium-selenium chalcogenide glasses" J. Am. Ceram. Soc. 85, 1545 (2002)

${ }^{43}$ T. G. Edwards, and S. Sen, "Structure and Relaxation in Germanium Selenide Glasses and Supercooled Liquids: A Raman Spectroscopic Study" J. Phys. Chem. B 115, 4307 (2011)

${ }^{44}$ I. M. Hodge, and A. R. Berens, "Effects of annealing and prior history on enthalpy relaxation in glassy polymers. 2. Mathematical modeling" Macromolecules 15, 762 (1982)

${ }^{45}$ R. Boehmer, K. L. Ngai, C. A. Angell, and D. J. Plazek, "Nonexponential relaxations in strong and fragile glass formers" J. Chem. Phys. 99, 4201 (1993)

${ }^{46}$ I. M. Hodge, "Adam-Gibbs formulation of nonlinear enthalpy relaxation" J. Non-Cryst. Solids 131-133, 435 (1991)

${ }^{47}$ A. R. Berens, and I. M. Hodge, "Effect of annealing and prior history on enthalpy relaxation in glassy polymers. 1. Experimental study on poly(vinyl chloride)" Macromolecules 15, 756 (1982)

${ }^{48}$ I. M. Hodge, and G. S. Huvard, "Effects of annealing and prior history on enthalpy relaxation in glassy polymers. 3. Experimental and modeling studies of polystyrene" Macromolecules 16, 371 (1983)

${ }^{49}$ I. M. Hodge, "Effects of annealing and prior history on enthalpy relaxation in glassy polymers. 4. Comparison of five polymers" Macromolecules 16, 898 (1983)

${ }^{50}$ I. M. Hodge, and A. R. Berens, "Effects of annealing and prior history on enthalpy relaxation in glassy polymers. 5. Mathematical modeling of nonthermal preaging perturbations" Macromolecules 18, 1980 (1985)

${ }^{51}$ I. M. Hodge, "Effects of annealing and prior history on enthalpy relaxation in glassy polymers. 6. Adam-Gibbs formulation of nonlinearity" Macromolecules 20, 2897 (1987)

${ }^{52}$ T. Grande, M. Ishii, M. Akaishi, S. Aasland, H. Fjellvåg, and S. Stølen, "Structural Properties of GeSe2 at High Pressures" Journal of Solid State Chemistry 145, 167 (1999)

${ }^{53}$ A. Zeidler, P. Chirawatkul, P. S. Salmon, T. Usuki, S. Kohara, H. E. Fischer, and W. S. Howells, "Structure of the network glass-former $\mathrm{ZnCl}_{2}$ : From the boiling point to the glass" J. Non-Cryst. Solids 407, 235 (2015)

${ }^{54}$ P. Lucas, G. J. Coleman, M. Venkateswara Rao, A. N. Edwards, C. Devaadithya, S. Wei, A. Q. Alsayoud, B. G. Potter, K. Muralidharan, and P. A. Deymier, "Structure of ZnCl2 Melt. Part II: Fragileto-Strong Transition in a Tetrahedral Liquid" J. Phys. Chem. B 121, 11210 (2017)

${ }^{55}$ P. K. Gupta, and J. C. Mauro, "Composition dependence of glass transition temperature and fragility. I. A topological model incorporating temperature-dependent constraints" J. Chem. Phys. 130, 094503/1 (2009)

${ }^{56}$ G. G. Naumis, "Energy landscape and rigidity" Phys. Rev. E 71, 026114/1 (2005)

${ }^{57}$ J. C. Mauro, Y. Yue, A. J. Ellison, P. K. Gupta, and D. C. Allan, "Viscosity of glass-forming liquids" Proc. Natl. Acad. Sci. U. S. A. 106, 19780 (2009) 
${ }^{58}$ J. E. K. Schawe, "A comparison of different evaluation methods in modulated temperature DSC" Thermochim. Acta 260, 1 (1995)

${ }^{59}$ A. Boller, C. Schick, and B. Wunderlich, "Modulated differential scanning calorimetry in the glass transition region" Thermochim. Acta 266, 97 (1995)

${ }^{60}$ J. E. K. Schawe, "Principles for the interpretation of modulated temperature DSC measurements. Part 1. Glass transition" Thermochim. Acta 261, 183 (1995)

${ }^{61}$ O. Bustin, and M. Descamps, "Slow structural relaxations of glass-forming Maltitol by modulated DSC calorimetry" J. Chem. Phys. 110, 10982 (1999)

${ }^{62}$ G. Yang, O. Gulbiten, Y. Gueguen, B. Bureau, J.-C. Sangleboeuf, C. Roiland, E. A. King, and P. Lucas, "Fragile-strong behavior in the $\mathrm{As}_{\mathrm{x}} \mathrm{Se}_{1-\mathrm{x}}$ glass forming system in relation to structural dimensionality" Phys Rev. B: Condens. Matter 85, 144107/1 (2012)

${ }^{63}$ T. K. Bechgaard, O. Gulbiten, J. C. Mauro, Y. Hu, M. Bauchy, and M. M. Smedskjaer, "Temperaturemodulated differential scanning calorimetry analysis of high-temperature silicate glasses" ACerS Bulletin 97, 31 (2018)

${ }^{64}$ L. Carpentier, S. Desprez, and M. Descamps, "From strong to fragile glass-forming systems: A temperature modulated differential scanning calorimetry investigation" Phase Transitions 76, 787 (2003) 